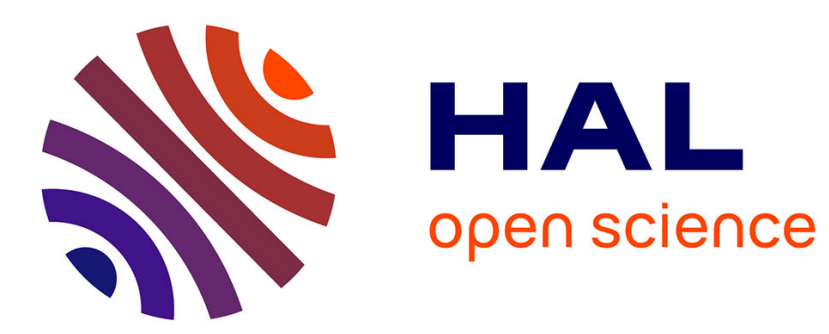

\title{
Orthogonal frequency division multiplexing-based time difference of arrival estimation
}

\author{
Ahmed Abudabbousa, Ammar Abu-Hudrouss, Aziz Benlarbi-Delai
}

\section{To cite this version:}

Ahmed Abudabbousa, Ammar Abu-Hudrouss, Aziz Benlarbi-Delai. Orthogonal frequency division multiplexing-based time difference of arrival estimation. IET Communications, 2019, 13 (15), pp.23222326. hal-02390485

\section{HAL Id: hal-02390485 \\ https://hal.sorbonne-universite.fr/hal-02390485}

Submitted on 26 Feb 2020

HAL is a multi-disciplinary open access archive for the deposit and dissemination of scientific research documents, whether they are published or not. The documents may come from teaching and research institutions in France or abroad, or from public or private research centers.
L'archive ouverte pluridisciplinaire HAL, est destinée au dépôt et à la diffusion de documents scientifiques de niveau recherche, publiés ou non, émanant des établissements d'enseignement et de recherche français ou étrangers, des laboratoires publics ou privés. 


\title{
OFDM Based Time Difference of Arrival Estimation
}

\author{
Ahmad Abudabbousa ${ }^{1 *}$, Ammar M. Abu-Hudrouss ${ }^{2}$, Aziz Benlarbi-Delai ${ }^{1}$ \\ ${ }^{1}$ Sorbonne Université, Laboratoire d'Électronique et Électromagnétisme, L2E, F-75005 Paris, France \\ ${ }^{2}$ Electrical Engineering Department, Engineering Faculty, Islamic University of Gaza, Gaza-Strip, Palestine \\ *E-mail: adabowsa@gmail.com
}

\begin{abstract}
This paper presents a spectral approach to estimate the time difference of arrival (TDOA) between a reference unit (RU), composed of a pair of very close antennas (small baseline regarding the operating bandwidth), acting as a known fixed location transmitter, and a mobile unit (MU) acting as a single antenna receiver. It uses the channel frequency response (CFR) based on an orthogonal frequency division multiplexing (OFDM) multicarrier communications in a multiple-input-single-output (MISO) antenna configuration. By handling the CFR responses, seen as wideband interferometric signals, we minimize a cost function expressed as the difference between measured channel response and a predefined direct model. Effects of noise and multipath are evaluated and mitigated by averaging process.

The overall performances of the system are analyzed, and the experimental validation is systematically led. In a small-scale indoor environment, it has been shown that the TDOA can be accurately estimated with higher accuracy than the conventional techniques. The proposed method allows, for example, estimating TDOAs of about 2 ns when using a null-to-null bandwidth of $100 \mathrm{MHz}$. Such an approach, based on existing communication systems, and suitable for the 5G norm, can be useful for several applications needing accurate positioning, without requiring complex dedicated infrastructure.
\end{abstract}

\section{Introduction}

The technique of time difference of arrival (TDOA) is a reciprocal method, which consists in measuring the differences of times of flight between a source and a known fixed location and synchronized measuring units. It can then help to solve a set of nonlinear equations to estimate the source location, meaning that the positioning problem is mainly seen as a time delay estimation (TDE) problem [1].

The classical TDE techniques are based on correlations [2]. However, correlation is computationally expensive with limited time resolution, and significantly degraded performance when the signal components are closely spaced. To deal with these restrictions, many high-resolution TDOA algorithms have been proposed and can be classified, in the time domain, into three branches. The first is the optimal maximal likelihood (ML) time delay estimators using techniques, e.g., expectation maximization (EM) [3], importance sampling [4], and formulating the time delay as a least squares (LS) fitting problem [5]. But, these algorithms often converge to a local optimum.

The second branch is based on the subspace methods that can explicitly cope with the multisource correlation matrix such as multiple signal classification (MUSIC) [6], estimation of signal parameters via rotational invariance techniques (ESPRIT) [7], and compressive sensing (CS) $[8,9,10]$. By observing the eigen-structure of the sensor correlation matrix, the signal subspace can be extracted and the TDOA can be estimated.

The third branch, mainly proposed for positioning issues, is the high resolution TDOA estimation methods using difference of convex functions (DC) programming based on the least-mean absolute, i.e., the $l_{1}$ norm, minimization of the residual errors $[11,12]$. A nonlinear weighted least squares (WLS) is introduced in [13] to avoid convergence to a local minima. In [14], quadratic programming is used for passive multi-satellite TDOA localization.

Except for these main branches, some delay estimation techniques, having moreover the ability to cope with multipath environment, can also be adapted to TDOA estimation. Hou and Wu proposed a model-based sinusoidal estimation method in [15], which converted time delay estimation problem into a sinusoidal parameter estimation problem. These methods often involve a spectral division operation and do not deal with the scenario where paths have close delays, as expected in small indoor environments.

On the other hand, the very popular OFDM technology ensuring intrinsic robustness to multipath, and accurate time management, is also a good candidate for precise TDOA estimation. OFDM technique, which is adopted worldwide in numerous well-known communication standards like: DVB-T, IEEE 802.11a/g, 802.11n, WiMAX, UWB, LTE/4G, may also help, by the way, solving positioning issue by exploring mainly two sets of approaches. The first uses the conventional or improved timing synchronization algorithms to locate the boundaries of OFDM signals [16]. The second category is based on super resolution algorithms such as maximum likelihood (ML), or matrix pencil (MP) derived from modern spectral estimation techniques [17]. The super resolution algorithm is applied to the frequency-domain channel estimation to extract a more accurate estimate of the first path in the time domain. To guard against the limitations mentioned above while exploiting existing communication systems, we present an OFDM based TDOA estimation, especially devoted to small delay differences with respect to the available frequency bandwidth. We formulate the problem, based on the estimation of channel state information (CSI), and state that the proposed approach has a main advantage over the LS-based methods $[6,7,11]$, where large numbers of samples are required. Both numerical simulations and experimental results evaluate the performance of the proposed TDOA estimator.

\section{Geometry of acquisition}

The scenario at the basis of the present work is described in Fig. 1 [17]. The adopted configuration is a multiple-input-single-output (MISO) configuration which is composed, using reciprocity principle, of a single known fixed location reference unit (RU) using a 


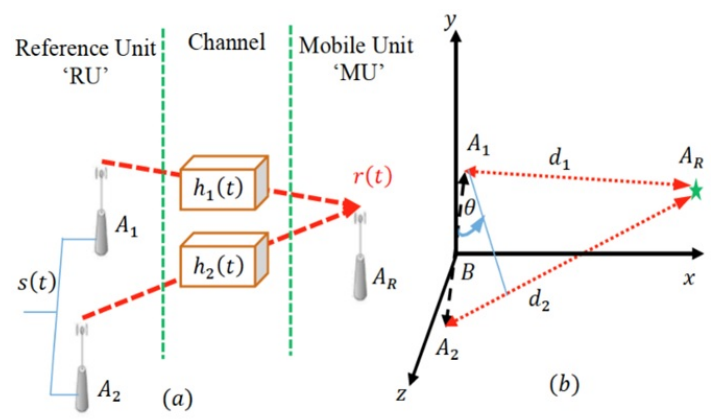

Fig. 1: (a) MISO configuration, (b) Associated geometry

radio frequency $(\mathrm{RF})$ source associated with two collocated transmit antennas $A_{1}$ and $A_{2}$ separated by a small baseline $B$, and a mobile unit (MU) with a single receiving antenna $A_{R}$. It is clear that close transmitting antennas solves the problem of the synchronization required for estimating the TDOA $\tau$ defined as:

$$
\tau=\frac{d_{2}-d_{1}}{c}
$$

where $c$ is the speed of light, $d_{1}$ and $d_{2}$ are the respective distances between nodes $A_{R}$ and $A_{1}$, and nodes $A_{R}$ and $A_{2}$, respectively. Let us note that the values of $\tau$ are bounded by the baseline $B$ and hence verify, $-\frac{B}{c} \leq \tau \leq \frac{B}{c}$. But for the sake of simplicity and for symmetry reasons, we consider, in this preliminary study, only the interval $\left[0, \frac{B}{c}\right]$.

\subsection{Signal Model}

The RU sends, periodically and simultaneously through antennas $A_{1}$ and $A_{2}$, an RF signal $s(t)$ toward MU. At the transmitter, we consider a data sequence $\left(X\left[-\frac{N}{2}\right], X\left[-\frac{N}{2}+1\right], \ldots, X\left[\frac{N}{2}-1\right]\right)$, where each $X[k]$ is drawn from QPSK constellation, and $N$ is the total number of OFDM subcarriers. These components are passed through Inverse Fast Fourier Transform (IFFT) block with added cyclic prefix, then up sampled at sampling frequency $f_{s}$ and applied, at the time intervals $T_{d}$, to a root raised cosine filter with a roll-off factor $\beta$. The resulting available bandwidth $f_{d}$ is then expressed as follow:

$$
f_{d}=\frac{1}{(1+\beta) T_{d}}
$$

and the resulting up sampled OFDM transmitted signal $s(t)$, centered around the carrier frequency $f_{c}$, can now be written as:

$$
s(t)=\Re\left\{x(t) e^{j 2 \pi f_{c} t}\right\}
$$

where $\Re$ stands for the real part and $x(t)$ is the continuous time version of the OFDM signal $x[n]$ which is given by [19]:eq

$$
x[n]=\frac{1}{\sqrt{N}} \sum_{k=-\frac{N}{2}}^{\frac{N}{2}-1} X[k] e^{\frac{j 2 \pi n k}{N}}, \quad-\frac{N}{2} \leq n \leq \frac{N}{2}-1
$$

At the receiver, the signal $r(t)$ which is affected by an additive white Gaussian noise (AWGN) $w(t)$ with zero mean and a variance of $\sigma_{n}^{2}$, can be written as:

$$
r(t)=h_{1}(t) * s(t)+h_{2}(t) * s(t-\tau)+w(t)
$$

where $(*)$ stands for convolution product, and $h_{1}(t)$ and $h_{2}(t)$ are the channel impulse responses respectively associated to link between nodes $A_{1}$ and $A_{R}$ and to link between nodes $A_{2}$ and $A_{R}$. The channel equalization and data demodulation can be simply done

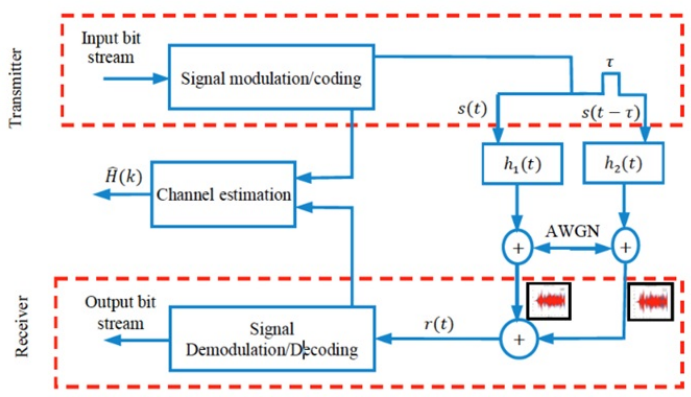

Fig. 2: Simulation model for a SISO system acting as a MISO system

after the Fast Fourier Transform (FFT) at the receiver via a bank of one-tap multipliers as:

$$
Y[k]=\left(H_{1}[k] X[k]+H_{2}[k] X[k] e^{\frac{j 2 \pi k \tau}{N}}\right)+W[k]
$$

where $-\frac{N}{2} \leq k \leq \frac{N}{2}-1, Y[k]$ is the FFT output of the down converted sampled version of $r(t)$ and $H_{1}[k]$ and $H_{2}[k]$ are the channel transfer functions associated respectively to $h_{1}(t)$ and $h_{2}(t) . W[k]$ is the sampled white noise generated within the subcarrier bandwidth with a noise power of $\left(\sigma_{n}^{2} f_{d} / N\right)$.

\subsection{Definition of the Direct Model}

As well known, the effect of the channel around the carrier frequency $f_{c}$ is integrally reproduced in baseband domain around $f=0 \mathrm{~Hz}$ and leads to help extracting the useful TDOA $\tau$ by using a direct model derived from (6). We consider the two following scenarios.

2.2.1 Free space scenario: Considering a free space scenario, the MISO direct model extracted from (6) is simply a two-path model channel transfer function expressed as:

$$
H[k, \tau]=\alpha_{1}+\alpha_{2} e^{\frac{-j 2 \pi k \tau}{N}}
$$

To estimate the coefficients $\alpha_{1}, \alpha_{2}$, we start by using a Single Input Single Output configuration (SISO) by feeding one antenna at a time. Assuming the knowledge of references pilot of the transmitted signal, thanks to OFDM technique, it becomes easy to determine, by exploiting the measured I-Q received signals, the complex transfer function of the channel. Anyway, this measurement does not need to be perfectly reproducible over a long period of time, but it is assumed that the channel remains unchanged during the switch time from SISO configuration to MISO configuration.

To emulate the effect of the TDOA on the channel response, we build up a SISO system as depicted in Fig. 2.

This system acts as a MISO configuration but without needing to take into account physically of the baseline $B$. Considering an input bit stream, the transmitted data - a random sequence using QPSK-OFDM modulation - is first split into two branches, and a controllable time delay $\tau$, playing the role of TDOA of interest, is inserted in one branch. In each branch modeled by its impulse response $h_{i}(t),(i=1,2)$, an AWGN is added. The combination of these two branches leads to the useful OFDM signal from which the channel estimation is performed at the receiving part.

Based on the parameter values stated in Table 1, Fig. 3 shows, for different TDOAs, the simulated CFR. Each curve is fitted by a function extracted from the MISO direct model (black dotted line).

As expected, these curves have a sinusoidal amplitude modulation of the spectrum, whose frequency period, which is naturally related to $\tau$, is noted $\delta f$.

2.2.2 Multipath scenario: To model the multipath, brought by Non-Line Of Sight (NLOS) propagation, we reformulate the 
Table 1 OFDM frame parameters

\begin{tabular}{lcc}
\hline Symbol & Parameters & Quantity \\
\hline$f_{d}$ & Bandwidth & $100 \mathrm{MHz}$ \\
$f_{s}$ & Sampling frequency & $10 \mathrm{GS} / \mathrm{s}$ \\
$f_{c}$ & Central frequency & $3 \mathrm{GHz}$ \\
$\beta$ & Roll-off factor & 0.35 \\
$N$ & \# of Sub-Carriers & 1024 \\
$\mu$ & Cyclic prefix length & 10 \\
$f_{P}$ & Pilots frequency & 1 \\
\hline
\end{tabular}

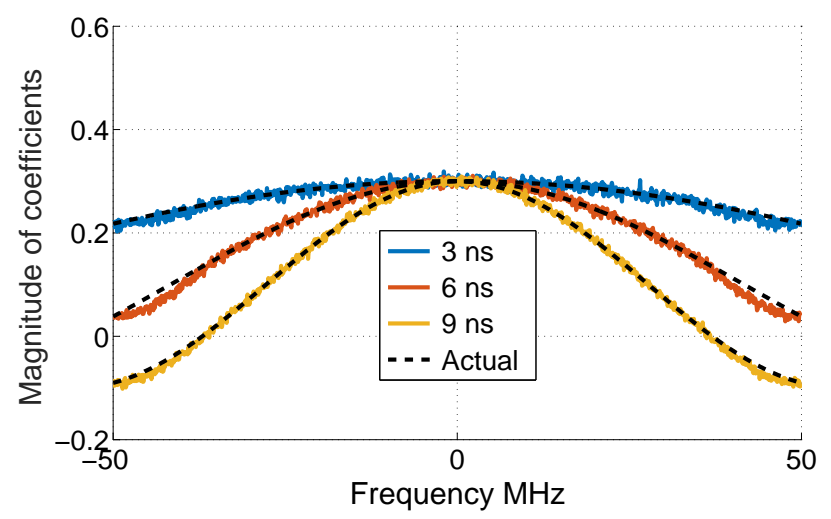

Fig. 3: Examples of the channel estimation obtained in MISO configuration with with $\mathrm{SNR}=20 \mathrm{~dB}$ : Test conditions are reported in Table 1

direct model defined in eq. (7) as a new channel transfer function $H_{M P}[k, \tau]$ that can be written as the sum of three terms:

$$
\begin{array}{r}
H_{M P}[k, \tau]=H[k, \tau]+\sum_{p=1}^{M} \beta_{p} e^{\frac{-j 2 \pi k \tau_{p}}{N}}+ \\
\sum_{q=1}^{L} \gamma_{q} e^{\frac{-j 2 \pi k \tau_{q}}{N}}
\end{array}
$$

The first term is the free space direct model, already presented, encompassing the useful TDOA, the second term refers with the $M$ parasitic contributions, weighted by $\beta_{p}$ and provided by multipath occasioning large TDOAs compared to $\tau$, and the third term refers with the $L$ parasitic contributions, weighted by $\gamma_{q}$ and provided by multipath occasioning small TDOA - i.e. that fall within the useful measurable range $\left[0, \frac{B}{c}\right]$.

OFDM with cyclic prefixes is a well-known solution that has been proven to be very efficient to mitigate multipath.

However, in non-classical situations, such as the adopted MISO configuration, new effects associated to the path difference between several multipaths occur and degrade the accuracy of the measurement. We investigate in the next session a new solution dealing with estimation of small TDOA in a noisy and multipath channel.

\section{The inverse problem: TDOA estimation}

Considering first a perfect scenario (no noise, no multipath), and once the coefficients $\alpha_{1}, \alpha_{2}$ in (7) duly estimated, the TDOA is easily extracted and expressed by:

$$
\tau=\frac{1}{\delta f}
$$

Let's note that if $\tau=\tau_{\max }$, the frequency period will be noted $\delta f_{\min }$ and set to $\frac{c}{B}$.
Solving the inverse problem by this way supposes that, for each estimated TDOA, the CFR exhibits at least one frequency period $\delta f$, assuming that $\delta f_{\min } \leq f_{d}$. This hypothesis is no longer verified when the TDOA is small with regard to the inverse of $f_{d}$. We meet this situation when the baseline $B$ is too small compared to $\frac{c}{f_{d}}$. For such a situation, no periodicity appears in the CFR and hence Fourier approach is no longer possible. This motivates the inverse problem solving we investigate in this section.

As we target small TDOA estimation, we need to overpass the physical limitation brought by Fourier resolution, but we can never exceed the physical limit brought by the frequency sampling $f_{s}=\frac{1}{T_{s}}$. Otherwise the smallest TDOA one can estimate must verify: $\tau \geq \frac{1}{f_{s}}$. However, as the received spectrum is first detected around $f_{c}$ and then down converted to base band signal, one must take care of the effect of $f_{c}$. To really make the up-converted spectrum (around $f_{c}$ ) behave truly like around $0 \mathrm{~Hz}$ - i.e. they are matched in phase- we estimate only TDOAs that are as close as possible to a multiple of $\frac{1}{f_{c}}$. This solution acts like a matched filter and avoid large error due to the phase mismatch. It also prevents from inaccuracy due to the effect of the roll off factor on the spectrum. On the other side, the greatest TDOA, that we suggest to solve, without using Fourier approach, should verify: $\tau<\tau_{\max }=\frac{1}{f_{d}}$. So, in practice the considered TDOA belongs to the interval $\left[\frac{1}{f_{s}}, \frac{1}{f_{d}}\right]$.

As we target inverse problem solving in noisy and multipath channel, the proposed solution is based on averaging process. Indeed, by integrating, over the available bandwidth $f_{d}$, the variable term of the measured channel transfer function, we obtain an unambiguous TDOA dependent function called useful information and noted $U S(\tau)$ (eq. 10). As a result, we reduce the effects of noise and multipath, specially those at the origin of parasitic large TDOAs.

$$
U S(\tau)=\frac{1}{N} \sum_{k=-\frac{N}{2}}^{\frac{N}{2}-1} H_{M P}[k, \tau]
$$

Therefore, solving the inverse problem consists in the minimization of a cost function defined by the distance between measured $U S(\tau)$ and a sinc function, brought by averaging over $f_{d}$ the normalized variable term of the direct model. The best value of $\tau$ should then verify:

$$
\tilde{\tau}=\operatorname{argmin}\left(U S(\tau)-\operatorname{sinc}\left(\pi \mathbf{T} f_{d}\right)\right)
$$

where $\mathbf{T}$ is a vector of TDOA candidates belonging to $\left[\frac{1}{f_{s}}, \frac{1}{f_{d}}\right]$ with, as suggested previously, a step time close to $\frac{1}{f_{c}}$. However averaging process allows reducing in (8) the term, which refers to the large parasitic TDOAs, but is not suitable with the term referring to small parasitic TDOAs, which are not dimmable by means of time discrimination approach. We suggest reducing their impact by means of power discrimination. Actually, due to the attenuation of propagation, multipath signals are naturally weaker than direct path, but one can enhance this effect by using circular polarization. It results a great reduction of the variation of RMS delay spread [20].

\subsection{Cramer Rao Lower Bound}

To evaluate the performance of the proposed solution we compare it to the optimal performance given by the Cramer Rao Lower Band (CRLB). This bound noted $\sigma_{C R}$, is extracted from Fisher information. If we consider the received signal power density spectrum having a rectangular shape, $\sigma_{C R}$ can be expressed, for TDOA estimation, as follow [21]:

$$
\sigma_{C R}=\frac{\sqrt{3}}{\pi f_{d}} \frac{1}{\sqrt{f_{d} T_{o b s} S N R_{I N}}}
$$

where $S N R_{I N}$ is the effective input signal to noise ratio, and $T_{o b s}$ is the observation time.Taking a fixed value of TDOA set for example to $0.7 \mathrm{~ns}, 100$ simulations have been performed with several observation times under several SNR values.

Fig. 4 shows that the standard deviation (STD) of the proposed 


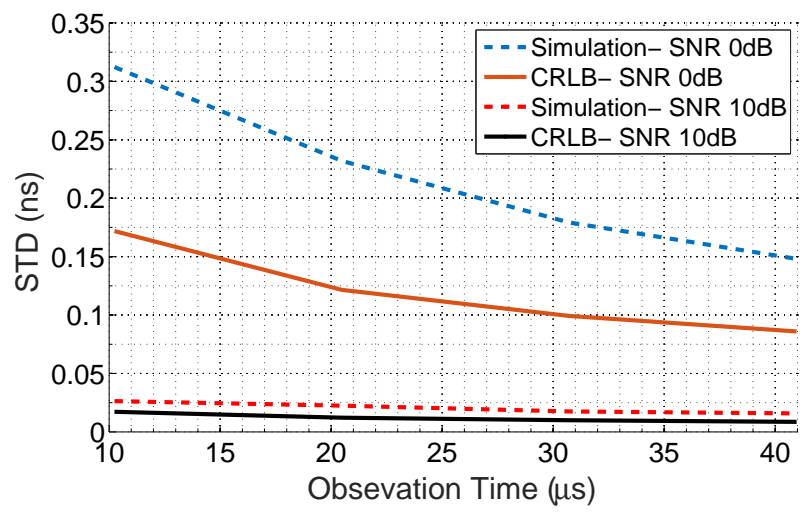

Fig. 4: CRLB Theory and simulation results comparison for $U S(\tau)$ based estimator: : test conditions are reported in Table 1

approach is asymptotic to CRLB and may attain it at higher SNR, making the proposed estimator more efficient.

\section{$4 \quad$ Results}

To verify the model described above and to test the inverse problem solving, simulation and experimental bench have been set up. The simulation set up is the SISO configuration presented in Fig. 2. The experimental environment is an anechoic chamber simulating free space scenario. TDOA of interest and parasitic TDOAs are emulated via MATLAB interface. Using the test parameters summarized in Table 1, the sounding OFDM frame (OF) is initially designed on MATLAB and replicated, via a controllable delay line. The resulting frames, which are now two delayed signals, are first sent to an arbitrary waveform generator (AWG) - $7 \mathrm{GHz}$ bandwidth and 24 $\mathrm{GS} / \mathrm{s}$ (when interleave mode is on). Two collocated identical antennas $A_{1}$ and $A_{2}$ - from SkyCross 222-1137B with a standing wave ratio $(\mathrm{SWR})<2.0$ and a maximum gain of $0.7 \mathrm{dBi}$ - transmit the output signals toward a receiver. For the air interface, a particular MISO situation is considered. Actually, the MU is facing the RU composed of antennas $A_{1}$ and $A_{2}$ meaning that $d_{1}$ and $d_{2}$ are perfectly equal and set to $2.4 \mathrm{~m}$. With such a scenario, the geometrical TDOA is set to 0 and will not impact the actual TDOA of interest defined and controlled by MATLAB. To reach suitable SNR, a power amplifier with output power, at $1 \mathrm{~dB}$ compression, of $13 \mathrm{dBm}$ and a low-noise amplifier of gain $33 \mathrm{~dB}$ and noise factor $\mathrm{NF}=3.5 \mathrm{~dB}$, are used respectively at the transmission and the reception parts.

At the receiving side, we collect, via a single antenna, identical to

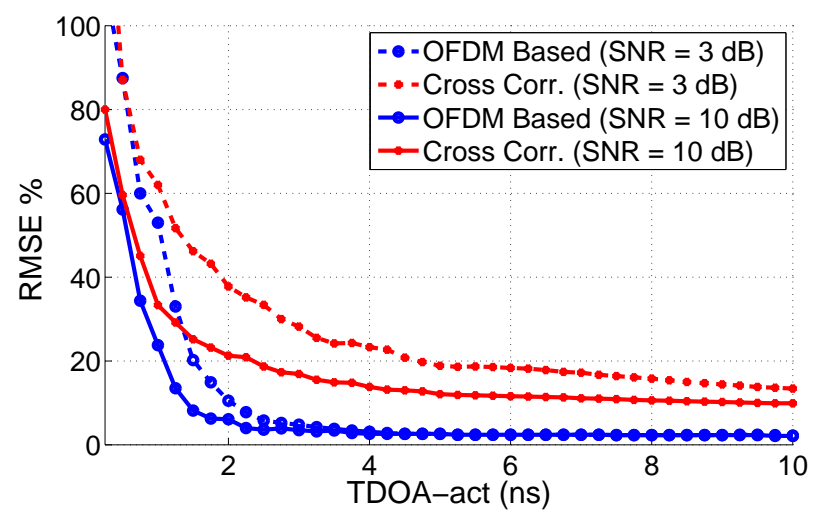

Fig. 5: Simulation results: the relative RMSE as a function of actual TDOA, for OFDM and cross correlation based methods for various SNR : test conditions are reported in Table 1

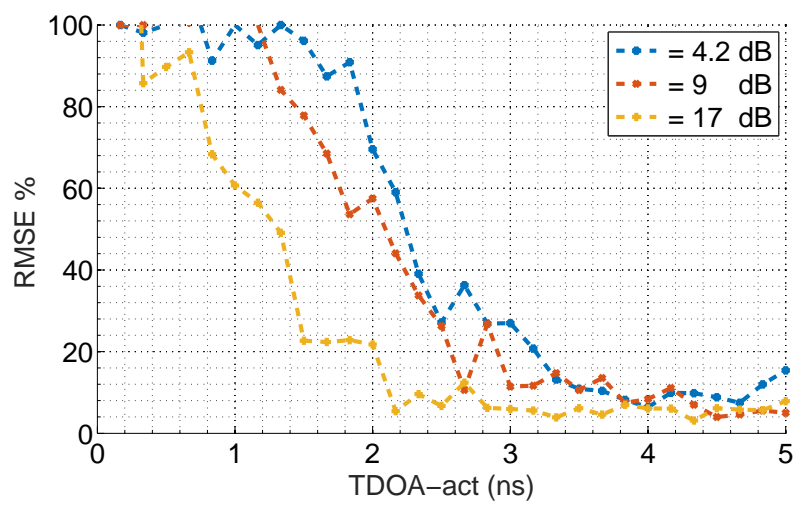

Fig. 6: Experimental results: the relative RMSE as a function of actual TDOA, for various SNR: test conditions are reported in Table 1

that of transmitting part, raw data on a complex signal analyzer with $12 \mathrm{GHz}$ bandwidth and $40 \mathrm{GS} / \mathrm{s}$. The received signal is processed according to the method mentioned above in order to estimate TDOA.

It can be seen from Fig. 5 that for the simulation part, the TDOA can be estimated with an error less than $10 \%$, down to $1.5 \mathrm{~ns}$ for SNR superior to $10 \mathrm{~dB}$, and down to $2 \mathrm{~ns}$ for SNR close to $3 \mathrm{~dB}$. The results indicate clearly the superiority of the introduced scheme compared with conventional cross correlation technique [22] that exhibits root mean square error (RMSE) less than $10 \%$, down to 4.75 ns for SNR superior to $10 \mathrm{~dB}$, and down to $9.5 \mathrm{~ns}$ for SNR close to $3 \mathrm{~dB}$.

The experimental results validate such an approach and it can be seen Fig. 6 that when SNR is greater than $17 \mathrm{~dB}$, an RMSE of less than $10 \%$ is obtained for TDOAs superior to $2 \mathrm{~ns}$. The result demonstrates that using an available bandwidth $f_{d}=100 \mathrm{MHz}$, the proposed approach makes possible to resolve TDOAs that normally require, for conventional approach, an equivalent bandwidth of $500 \mathrm{MHz}$.

But, as clearly shown in Fig. 5 and Fig. 6, the proposed solution fails in estimating very small TDOA, typically belonging to $\left[\frac{1}{f_{s}}, \frac{1}{10 f_{d}}\right]$. In practice, this situation occurs when the MU is facing the RU, we talk about paraxial situation meaning that $d_{1}$ and $d_{2}$ are very close and then the TDOA is close to 0 . This is due to the shape of the sinc function in (11) where, for very small TDOAs, the slope is quite null, leading to a weak sensitivity.

We present in the next paragraph a way to solve this drawback and treat also the way of mitigating small parasitic TDOAs.

\subsection{Improvement suggestions}

4.1.1 Very small TDOA estimation: To make the estimation of very low TDOA more accurate, we need to explore the sinc function in (11) and to detect the zone where the slope is high. That means that the sensitivity of the estimation is better when the useful TDOAs are in the vicinity of this zone. So, to make very small TDOAs belonging to this zone, one should add in one branch of the scenario depicted in Fig. 2 a delay line, which time delay noted $\tau_{\text {opt }}$ verifies:

$$
\left.\frac{d^{2}}{d \tau^{2}} \operatorname{sinc}\left(\pi f_{d} \tau\right)\right|_{\tau=\tau_{o p t}}=0
$$

Taking into account the test conditions reported in Table $1, \tau_{o p t}$ is set to $4.5 \mathrm{~ns}$. After insertion of such a delay line, we show Fig. 7 that the proposed improvement exhibits, as expected, better performance, for very small TDOAs.

It seems also that the different levels of noise power do not affect significantly the estimator performance, by virtue of the averaging process. 


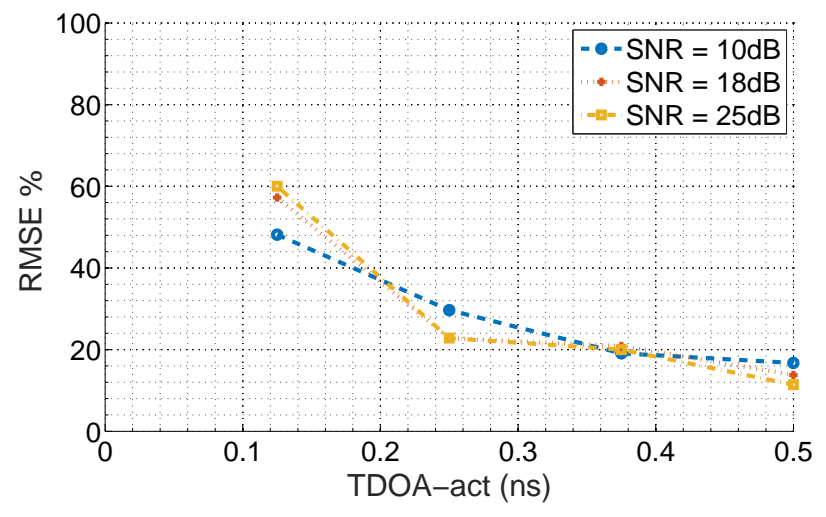

Fig. 7: Estimator performance with $\tau_{o p t}=4.5 \mathrm{~ns}$ the relative RMSE as function of the actual TDOA: test conditions are reported in Table 1

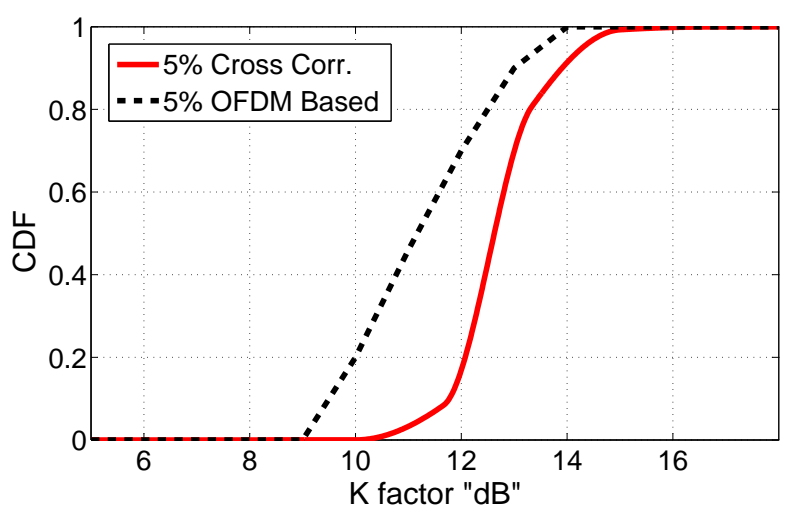

Fig. 8: Fitted Normal Cumulative Distribution Function (CDF) with $5 \%$ accepted error of the estimated value $\left(\tau_{o p t}=4.5 \mathrm{~ns}\right.$, with SNR $=$ $20 \mathrm{~dB})$. Test conditions are reported in Table 1

4.1.2 Small parasitic TDOAs mitigation: To investigate this effect, we performed simulation considering multipaths having random delays with average of $1.2 \mathrm{~ns}$ and variance of 0.0825 meaning that they fall within the TDOA range of interest. The total power of multipath is defined through the Rician factor $\mathrm{K}$, for which high values, particularly provided by using circular polarization antenna, mean that LOS components are more dominant than NLOS components.

Fig. 8 shows the cumulative distribution function (CDF) of the accuracy obtained on the estimated TDOA from simulation with 100 iterations. It can be observed that, assuming a SNR $=20 \mathrm{~dB}$, the error induced by multipath giving small parasitic TDOAs is always less than 5\% with K factor larger than $14 \mathrm{~dB}$. Superiority over conventional cross-correlation technique is hence established.

This power-based solution seems to work good enough and may improve the performance of the proposed solution in a realistic channel. Moreover, as the proposed solution targets millimetre wave scenario envisaged in the $5 \mathrm{G}$ networks, directional communication and suitable beamforming can also contribute to increase $\mathrm{K}$ factor and hence to spatially discriminate multipath components.

\section{Conclusion}

In this paper a spectral method, well suited with OFDM communication format, is proposed to extract TDOA metric from channel frequency response. This approach, working in noisy and multipath environment, exploits the channel state information and allows resolving time delays as small as one-fifth of the inverse of the available bandwidth. The simulation results, validated by several experiments, demonstrate the super-resolution character of such a solution.

It should be noted that with abundant bandwidth offered by $5 \mathrm{G}$ networks and the location function as an integral part of this upcoming standard, much lower time delays, less than few hundreds of picosecond could be measured without requiring large baseline, leading to very accurate indoor positioning system.

\section{Acknowledgments}

This work was supported by the European PHOENIX project and the doctoral institute of Sorbonne University. The authors would like to thank Zamala program and Bank of Palestine for supporting a short research visit to the L2E. We also would like to acknowledge the time and effort devoted by reviewers to improving the quality of this paper.

\section{References}

[1] Blandin, C., Ozerov, A., and Vincent, E.: 'Multi-source TDOA estimation in reverberant audio using angular spectra and clustering', Signal Processing, 2012, 92, (8), pp. 1950-1960

[2] Shi, H., Zhang, H., and Wang, X.: 'A TDOA technique with super-resolution based on the volume cross-correlation function' IEEE Transactions on Signal Processing, 2016, 64, (21), pp. 56825695

[3] Cui, X., Yu, K., and Lu, S.: 'direction finding for transient acoustic source based on biased TDOA measurement', IEEE Transactions on Instrumentation and Measurement, 2016, 65, (11), pp. 2442 2453

[4] Masmoudi, A., Bellili, F., Affes, S., and Stephenne, A.: 'A maximum likelihood time delay estimator in a multipath environment using importance sampling', IEEE Transactions on Signal Processing, 2013, 61, (1), pp. 182-193

[5] Yu, H., Huang, G., Gao, J., and Liu, B.: 'An efficient constrained weighted least squares algorithm for moving source location using TDOA and FDOA measurements', IEEE Transactions on Wireless Communications, 2012, 11, (1), pp. 44-47

[6] Liu, L. and Liu, H.: 'Joint estimation of DOA and TDOA of multiple reflections in mobile communications', IEEE Access, 2016, 4, pp. 3815-3823

[7] Fayad, Y., Wang, C., and Cao, Q.: 'Temporal-spatial subspaces modern combination method for 2D-DOA estimation in MIMO radar', Journal of Systems Engineering and Electronics, 2017, 28, (4), pp. 697-702

[8] Salari, S., Chan, F., Chan, Y.-T., and Read, W.: 'TDOA estimation with compressive sensing measurements and Hadamard matrix', IEEE Transactions on Aerospace and Electronic Systems, 2018, 54, (6), pp. 3137-3142

[9] Cao, H., Chan, Y. T., and So, H.-C.: 'Maximum likelihood TDOA estimation from compressed sensing samples without reconstruction', IEEE Signal Processing Letters, 2017, 24, (5), pp. 564568

[10] Skiribou, C., Elassali, R., Boukour, F., Elbaamrani, K., Idboufker, N., Benlarbi-Delai, A., 'LOS Identification for high accuracy TOA-based ranging system in tunnel environments', AEÜ International Journal of Electronics and Communications, 2019, 98 , pp. 38-44

[11] Gholami, M. R., Gezici, S., and Strom, E. G.: 'A concaveconvex procedure for TDOA based positioning', IEEE Communications Letters, 2013, 17, (4), pp. 765-768

[12] Su, Z., Shao, G., and Liu, H.: 'Semidefinite programming for NLOS error mitigation in TDOA localization', IEEE Communications Letters, 2018, 22, (7), pp. 1430-1433

[13] Wang, G., Cai, S., Li, Y., and Ansari, N.: 'A bias-reduced nonlinear WLS method for TDOA/FDOA-based source localization', IEEE Transactions on Vehicular Technology, 2016, 65, (10), pp. 8603-8615 
[14] Shu, F., Yang, S., Qin, Y., and Li, J.: 'Approximate analytic quadratic-optimization solution for TDOA-based passive multisatellite localization with earth constraint', IEEE Access, 2016, 4, pp. 9283-9292

[15] Zi-qiang., H., and Zhen-dong, W.: 'A new method for high resolution estimation of time delay'. Proc. IEEE Int. Conf. on Acoustics, Speech, and Signal Proc. (ICASSP'82), Paris, France, May 1982, pp. 420-423

[16] Martin, R. K., Yan, C., Fan, H. H., and Rondeau, C.: 'Algorithms and bounds for distributed TDOA-based positioning using OFDM signals', IEEE Transactions on Signal Processing, 2011, 59, (3), pp. 1255-1268

[17] Khanzada, T. J., Ali, A. R., and Omar, A. S. : 'Time difference of arrival estimation using super resolution algorithms to minimize distance measurement error for indoor positioning systems'. Proc. IEEE int. Multitopic Conf., Karachi , Pakistan, Dec. 2008, pp. 443447

[18] Abudabbousa A., Sarrazin J., De Doncker P., Benlarbi-Delaï1 A.: 'Multicarrier technique-based super-resolution TDOA estimation'. Proc. Int. Conf. RF/microwave and antenna technologies, Marseille, France, Nov. 2017

[19] Goldsmith, A.,: 'Wireless communications' Cambridge university press, 1st Edition. 2005.

[20] Rappaport, T. S., Hawbaker, D. A. 'Wide-band microwave propagation parameters using circular and linear polarized antennas for indoor wireless channels', IEEE Transactions on Communications, 1992, 40, (2), pp. 240-245

[21] Stein, S.: 'Algorithms for ambiguity function processing', IEEE Transactions on Acoustics, Speech, and Signal Processing, 1981, 29, (3), pp. 588-599

[22] Seuté, H., Enderli, C.; Grandin, J.-F., et al.:'Experimental measurement of time difference of arrival'. Proc. 17th Int. Radar Symp. (IRS), Krakow, Poland, 2016 